Schmp. 182 ${ }^{\circ}$. Leicht löslich in Alkohol und Eisessig, kaum in Benzol und Ligroïn.

$0.1953 \mathrm{~g}$ Sbst.: $18 \mathrm{ccm} \mathrm{N}\left(18^{\circ}, 778 \mathrm{~mm}\right)$.

$\mathrm{C}_{15} \mathrm{H}_{14} \mathrm{O}_{2} \mathrm{~N}_{2}$. Ber. $\mathrm{N} 11.03$. Gef. $\mathrm{N} 10.91$.

Diacetylverbindung des $p$-Oxybenzaldehydphenylhydrazons, aus Benzol mit Ligroïn in linsenförmigen Aggregaten, die bei $148^{\circ}$ schmelzen.

0.0890 g Sbst.: $7 \mathrm{ccm} \mathrm{N}\left(16^{0}, 772 \mathrm{~mm}\right)$. $\mathrm{C}_{17} \mathrm{H}_{16} \mathrm{O}_{3} \mathrm{~N}_{2}$. Ber. N 9.46. Gef. N 9.32.

Den Höchster Farbwerken bin ich für die gütige Ueberlassung des Aldehyds zu grossem Danke rerptichtet.

Greifswald, chemisches Institut.

674. M. Rogow: Ueber Dialdehyde, welche durch Einwirkung von Aldehyden auf aromatische Oxyaldehyde entstehen.

[III. Mittheilung:

Ueber $p$ - a ad $m$-Nitrobenzaldimethyldivanillin.]')

(Eingegangen am 23. November 1903.)

In den von mir in der zweiten Mittheilung beschriebenen $p$ - und $m$-Nitrobenzal-divanillinen,

$$
\mathrm{NO}_{2} . \mathrm{C}_{6} \mathrm{H}_{4} \cdot \mathrm{CH}\left[\mathrm{C}_{6} \mathrm{H}_{2}(\mathrm{OH})\left(\mathrm{OCH}_{3}\right)(\mathrm{CHO})\right]_{2} \text {, }
$$

lassen sich beide Hydroxylwasserstoffatome durch Methylgruppen ersetzen. Die so entstehenden Körper sind als Condensationsproducte von 1 Molekül $p$ - bezw. $m$-Nitrobenzaldehyd mit 2 Molekülen Protocatechualdehyddimethyläther aufzufassen:

$$
\begin{array}{r}
\mathrm{NO}_{2} \cdot \mathrm{C}_{6} \mathrm{H}_{4} \cdot \mathrm{CHO}+2 \mathrm{C}_{6} \mathrm{H}_{3}\left(\mathrm{OCH}_{3}\right)_{2}(\mathrm{CHO}) \\
=\mathrm{NO}_{2} \cdot \mathrm{C}_{6} \mathrm{H}_{4} \cdot \mathrm{CH}\left[\mathrm{C}_{6} \mathrm{H}_{2}\left(\mathrm{OCH}_{3}\right)_{2}(\mathrm{CHO})\right]_{2}+\mathrm{H}_{2} \mathrm{O},
\end{array}
$$

und würden demgemäss als $p$ - und $m$-Nitrobenzal-bisprotocatechualdehyddimethyläther zu bezeichnen sein; um aber weniger schleppende Namen zu gebrauchen, nenne ich sie $p$ - und $m$-Nitrobenzal-dimethyldivanillin.

$$
p \text {-Nitrobenzal-dimethyldivanillin. }
$$

$15 \mathrm{~g} \mathrm{p}$-Nitrobenzal-diranillin $+100 \mathrm{ccm}$ einer Lösung ron $30 \mathrm{~g}$ Aetzkali in $800 \mathrm{ccm}$ Methylalkobol $+9 \mathrm{ccm}$ Methyljodid wurden in einen Kolben eingebracht und 5 Stunden in einem heissen Wasser-

1) Die beiden ersten Mittheilnagen: diese Berichte 34, 3881 [1901]; 35, 1961 [1902]. 
bade zum Sieden unter Rückfloss erbitzt. Dann wurde der Rückflusskühler entfernt und Wasser himzugesetzt, der Methylalkobol und dis überschüssige Methyljodid möglichst weggedampft, der Kolben abgekühlt und der Inhalt abgesaugt. Der abgesaugte Niederschlag wurde in ziemlich viel heissem Eisessig aufgenommen, eventuell von etwas ungelöster $\mathrm{Sub}$ stanz abfiltriıt und die Lösung in viel Wasser unter Umrühren hineingegossen. Dabei fiel ein Niederschlag aus, der am nächsten Tage abgesaugt und eine halbe Stunde in kochendem Wasserbade mit 5-proc. Natronlange erwärmt wurde. Nach dem $A$ bkühlen wurde abgesaugt, wieder in Eisessig gelöst, Thierkohle hinzugesetzt, eine balbe Stunde zum Sieden erhitzt, heiss filtrirt, das Filtrat in Wasser gegossen, der ausgefallene Niederschlag am nächsten Tage abgesaugt, mit rerdünnter Natronlauge eine halbe Stunde erwärmt, abgekühlt, abgesaugt und mit Wasser gewaschen. In diesem Zustande ist der Körper nach dem Trocknen für weitere Verarbeitungen genügend rein. Um ihn àalysenrein zu gewinuen, kochte man ihn mit nicht zuviel Methylalkohol aus, löste den ausgekochten Niederschlag in ziemlich riel heissem Alkohol und rersetzte ihn mit ungefähr dem doppelten Volumen beissen Wassers. Beim Erkalten schieden sich Krystalle aus, die dana auf dieselbe Weise noch ein Mal umbrystallisirt wurden. Für die Analyse wurde der Körper 2 Stunden im Toluolbade getrocknet.

$$
\begin{aligned}
& 0.2652 \mathrm{~g} \text { Sbst.: } 0.6259 \mathrm{~g} \mathrm{CO}_{2}, 0.1131 \mathrm{~g} \mathrm{H}_{2} \mathrm{O} . \\
& \mathrm{C}_{23} \mathrm{H}_{23} \mathrm{O}_{8} \mathrm{~N} \text {. Ber. C } 64.49, \mathrm{H} 4.98 . \\
& \text { Gef. } \$ 64.37 \text {, 》 } 4.77 .
\end{aligned}
$$

Der farblose Körper schmilzt bei $186-188^{\circ}$ (corr.) und verwandelt sich dabei in eine gelbliche Flüssigkeit; er bildet, aus verdünntem Alkohol krystallisirt, mikroskopische Prismen, die zuweilen an den Enden gezackt sind; er ist leicht löslich in Aceton, Chloroform, Essigester, schwerer in Benzol und heissem Alkobol, schwer löslich in heissem Aether und heissem Ligroün; eine Lösung des Körpers in concentrirter Schwefelsäure ist grünlich-gelb gefärbt; Fehling'sche Lösung, mit dem Körper 5 Minuten zum Kochen erhitzt, wird nicht reducirt; eine ammoniakalische Silberlösung, nach Tollens dargestellt, wird bei längerem Steben mit dem Körper im Dunkeln nursehr schwach reducirt.

p-Nitrobenzal-dimethyldiranillin-tetraacetat, $\mathrm{NO}_{2}, \mathrm{C}_{6} \mathrm{H}_{4} \cdot \mathrm{CH}\left[\mathrm{C}_{6} \mathrm{H}_{2}\left(\mathrm{OCH}_{3}\right)_{2} \cdot \mathrm{CH}\left(\mathrm{O} . \mathrm{CO} . \mathrm{CH}_{3}\right)_{2}\right]_{2}$.

3 Tropfen concentrirter Schwefelsäure wurden in $3 \mathrm{ccm}$ Essigsäureanhydrid gelöst und diese Lösung zu einem Gemisch von $2.7 \mathrm{~g}$ $p$-Nitrobenzal-dimethyldivanillin mit $7 \mathrm{~cm}$ Essigsäureanbydrid binzugefügt. Es findet starke Erwärmung statt. Die Masse wurde ge- 
schüttelt, bis sie anfing, sich in einen festen Brei zu rerwandeln, was nach ungefähr 2 Minuten der Fall war. Dann wurde mit viel Wasser versetzt, am nüchsten Tage abgesaugt, mit $30 \mathrm{ccm}$ Alkohol ausgekocht, der ausgekochte Niederschlag nach dem Absaugen in heissem Essigester gelöst, die Lösung auf $3 / 4$ ihres Volumens eingedampft, abgekühlt und mit Petroläther gefällt. . Der ausgefallene Niederschlag wurde noch ein Mal in heissem Essigester gelöst und die Lösung nach einigem Eindampfen und Erkaltenlassen mit Petroläther gefällt. Nach dem Absaugen and Waschen mit Petroläther wurde $1 \frac{1}{2}$ Stunden im Toluolbade getrocknet und analysit.

$0.2063 \mathrm{~g}$ Sbst.: $0.4448 \mathrm{~g} \mathrm{CO}_{2}, 0.1054 \mathrm{~g} \mathrm{H}_{2} \mathrm{O}$.

$$
\begin{aligned}
& \mathrm{C}_{33} \mathrm{H}_{35} \mathrm{O}_{14} \mathrm{~N} \text {. Ber. C 59.17, H } 5.27 \text {. } \\
& \text { Gef. " 58.50, " } 5.71 .
\end{aligned}
$$

Der schneeweisse, voluminöse Körper bildet verfilzte Nadeln, ist leicht löslich in Aceton and Chloroform, schwerer in Essigester, schwer löslich in heissem Benzol, heissem Alkobol und beissem Aether; er schmilat bei $186-188^{\circ}$ (corr.), also bei derselben Temperatur, bei der auch $p$-Nitrobenzal-dimethyldivanillin schmilzt; die beiden addirten Essigsäureanhydridmoleküle üben keinen merklichen Einfuss auf die Cohmelztemperatur aus.

\section{m-Nitrobenzal-dimethyldivanillin 1).}

In rohem Zustande wurde der Körper, wie oben beim $p$-Nitrobenzal-dimethyldivanillin beschrieben, dargestellt. Behufs Gewinnung analysenreiner Substanz wurde der Körper, nachdem er zum zweiten Male mit Natronlauge erwärmt war, aus heisser, 50-procentiger Essigsäure umkrystallisirt und dann in ziemlich viel heissem Alkohol gelöst und mit heissem Wasser bis zur schwachen Trübung rersetzt. Die

1) Anmerkung. Um m-Nitrobenzal-divanilin als Ausgangsmatcrial mit besserer Ausbeute und bequemer gewinnen zu können, wurde die früher angegebene Darstellungsweise abgeändert: $80 \mathrm{~g}$ Vanillin $+40 \mathrm{~g} \mathrm{~m}$-Nitrobenzaldehyd $+40 \mathrm{~g}$ frisch geschmolnenes Zinkchlorid warden $38-48$ Stunden auf 130-1460 erhitzt, die fest gebackene Masse nach Zertrümmerung des Erhitzungegefässes, fein zerrieben, mit $100 \mathrm{ccm}$ Alkohol ausgekocht, mit $100 \mathrm{ccm}$ Eisessig in kocbendem Wasserbade erwärmt, abgekühlt, abgesangt, dann wieder mit $75 \mathrm{ccm}$ Eisessig erwärmt, abgekühlt, abgesaugt, mit Alkohol gewaschen und bis zum constanten Gewicht getrocknet. Die Ausbente an $m$-Nitrobenzal-divanillin, das nur 2-2.50 niedriger als die analysen reine Substanz schmolz, schwankte bei 7 verschiedenen Darstellungen zwischen 70 und $87 \mathrm{~g}$ und war im Durchschnitt $77^{+/ 7} \mathrm{~g}$.

Auch beim $p$-Nitrobenzal-diranillin lässt sich die Ausbeute durch unwesentliche Abänderungen des früher mitgetheilten Verfahrens steigern. 
beim Erkalten ausgeschiedenen Krystalle wurden im Toluolbade 2 Stunden getrocknet und analysirt.

$0.2451 \mathrm{~g}$ Sbst.: $0.5803 \mathrm{~g} \mathrm{CO}_{3}, 0.1150 \mathrm{~g} \mathrm{H}_{2} \mathrm{O}$.

$$
\begin{aligned}
& \mathrm{C}_{25} \mathrm{H}_{23} \mathrm{O}_{8} \mathrm{~N} \text {. Ber. C 64.49, H 4.98. } \\
& \text { Gef. 》 } 64.57 \text {, } 5.25 \text {. }
\end{aligned}
$$

Der Körper schmilzt bei $181-183^{\circ}$ (corr.), ist leieht löslich in Aceton, Chloroform, Essigester, schwer löslich in heissem Benzol, heissem Alkohol und heissem Aether; aus verdünntem Alkohol krystallisirt er in sechsseitigen, mikroskopischen Prismen; in concentrirter Schwefelsäure ist er mit grünlich-gelber Farbe löslich; Tollens'sche Silberlösung reducirt der Körper bei längerem Stehen ganz unbedeutend; gegen Fehling'sche Lösung verhält er sich wie $p$-Nitrobenzaldimethyldivanillin.

Bis-phenylhydrazon des m-Nitrobenzal-dimethyldivanillins, $\mathrm{NO}_{2} \cdot \mathrm{C}_{6} \mathrm{H}_{4} \cdot \mathrm{CH}\left[\mathrm{C}_{6} \mathrm{H}_{2}\left(\mathrm{OCH}_{3}\right)_{2} \cdot\left(\mathrm{CH}: \mathrm{N} \cdot \mathrm{NH} \cdot \mathrm{C}_{6} \mathrm{H}_{6}\right)\right]_{2}$.

$2 \mathrm{~g}$ des $m$-Nitrobenzal-dimethyldivanillins wurden bei Siedehitze in $60 \mathrm{ccm}$ Eisessig gelöst, die Lösung beiss filtrirt und mit $3 \mathrm{ccm}$ Phe. nylhydrazin versetzt. Es wurde 2 Tage stehen gelassen, dann die abgeschiedenen Krystalle noch ein Mal aus heissem Eisessig umkrystallisirt, abgesaugt, mit Wasser gewaschen, 2 Stunden im Toluolbade getrocknet und analysirt. Für die Stickstoff bestimmang wurde der Körper mit Aether gewaschen und eine Stunde im Toluolbade getrocknet.

$0.2540 \mathrm{~g}$ Sbst.: $0.6358 \mathrm{~g} \mathrm{CO}_{2}, 0.1293 \mathrm{~g} \mathrm{H}_{2} \mathrm{O} .-0.1907 \mathrm{~g}$ Sbst.: $18.4 \mathrm{ecm}$ N $\left(14^{0}, 724 \mathrm{~mm}\right)$.

$$
\begin{aligned}
& \mathrm{C}_{37} \mathrm{H}_{35} \mathrm{O}_{6} \mathrm{~N}_{5} \text {. Ber. C 68.78, H 5.46, N } 10.88 \text {. } \\
& \text { Gef. » } 68.27, \gg 5.69, 》 10.78 \text {. }
\end{aligned}
$$

Der Körper ist gelb gefärbt, krystallisirt aus Eisessig in mikroskopischen Nadeln, schmilzt bei $203.5-204.5^{\circ}$ (corr.), ist leicht löslich in Aceton, Chloroform, Essigester, schwer löslich in heissem Benzol, beissem Alkohol und heissem Aether.

Die Untersuchung wird fortgesetzt.

München, November 1903. 